\title{
Global attractors of the periodic initial value problem for Landau-Lifshitz-Bloch-Maxwell system
}

Boling Guo ${ }^{1}$, Yitong $\mathrm{Pei}^{2}$, and Nan $\mathrm{Liu}^{3}$

${ }^{1}$ Institute of Applied Physics and Computational Mathematics

${ }^{2}$ Nanjing University of Science and Technology

${ }^{3}$ The Graduate School of China Academy of Engineering Physics

August 8, 2020

\begin{abstract}
This paper is devoted to study the global attractors of the periodic initial value problem for Landau-Lifshitz-Bloch-Maxwell system. Fist we give the global existence of the smooth solution for this system. Then, we prove the existence of global attractors, the Hausdorff dimension and fractal dimension have been estimated.
\end{abstract}

\section{Hosted file}

Global-attractors.pdf available at https://authorea.com/users/315233/articles/474631-globalattractors-of-the-periodic-initial-value-problem-for-landau-lifshitz-bloch-maxwellsystem 